\title{
Micro/Nanoscale Patterning of Nanostructured Metal Substrates for Plasmonic Applications
}

\author{
S. Shiv Shankar, Loris Rizzello, Roberto Cingolani, Ross Rinaldi, and Pier Paolo Pompa* \\ National Nanotechnology Laboratory of CNR-INFM, IIT Research Unit, Via Arnesano 16, 73100 Lecce, Italy
}

$\mathrm{R}$ ecent advances in understanding the surface properties of materials as well as in nanofabrication techniques have led to a considerable surge in attempts to control the structure, shape, and composition of surfaces at the nanoscale. The ability to tailor the surface structure has proven to be crucial for a wide variety of applications, including the improvement of the physiocompatibility of biomedical implants, ${ }^{1}$ the increase of the efficiency of electrodes for sensors and actuators, ${ }^{2,3}$ the achievement of enhanced catalytic properties, ${ }^{4}$ the maximization of superhydrophobicity and superhydrophilicity. 5,6 One of the most outstanding and novel outcomes of controlling the surface structure, especially of noble metals, is the possibility to engineer the spectral properties of certain molecules/fluorophores positioned in its vicinity, thanks to plasmonic coupling. Such technique, usually referred to as metal-enhanced fluorescence (MEF) ${ }^{7,8}$ may result in a remarkable increase of fluorophore emission intensity and has applications ranging from biodiagnostics to imaging, sensing, and photovoltaic cells. ${ }^{9,10}$

Owing to the importance associated with nanostructured surfaces, a number of experimental methods have been developed to date to fabricate such metallic surfaces. Some of the methods include simple thermally evaporated metal films, ${ }^{11}$ photoinduced deposition, ${ }^{12,13}$ electrochemical roughening of metal surfaces,,$^{14}$ chemical deposition of metals, ${ }^{11}$ adsorption of metal colloids on substrates, ${ }^{15}$ and dealloying.${ }^{16}$ In spite of these developments, there still remains enormous scope to apply new methods that can cater to the need of efficiently and precisely controlling the surface structure. In this respect, the essential require-
ABSTRACT The ability to precisely control the pattern of different metals at the micro- and nanoscale, along with their topology, has been demonstrated to be essential for many applications, ranging from material science to biomedical devices, electronics, and photonics. In this work, we show a novel approach, based on a combination of lithographic techniques and galvanic displacement reactions, to fabricate micro- and nanoscale patterns of different metals, with highly controlled surface roughness, onto a number of suitable substrates. We demonstrate the possibility to exploit such metal films to achieve significant fluorescence enhancement of nearby fluorophores, while maintaining accurate spatial control of the process, from submicron resolution to centimeter-sized features. These patterns may be also exploited for a wide range of applications, including SERS, solar cells, DNA microarray technology, hydrophobic/hydrophilic substrates, and magnetic devices.

KEYWORDS: metal patterning $\cdot$ nanostructure $\cdot$ gold $\cdot$ silver $\cdot$ metal-enhanced fluorescence

ments are the capabilities to achieve desired and accurate level of nanoroughness, extended uniformly over wide areas, while maintaining the ability to attain this in specific patterned regions, with micrometer or submicrometer spatial resolution.

We previously demonstrated that, by using electron beam lithography, it is possible to fabricate highly controlled patterns of gold nanoclusters with desired geometry and tunable optical properties that gave us the leverage to properly optimize the metal-enhanced fluorescence of colloidal nanocrystals. ${ }^{17}$ However, while this approach allowed us to obtain optimal MEF in desired patterns, it heavily relies on the complex and time-consuming fabrication of individual nanodimensional structures in the specific regions. In this report, we show that spontaneous galvanic displacement reactions (SGDR) in combination with lithographic techniques are a very efficient and promising strategy to fabricate spatially controlled two-dimensional patterns of one, two, or more different metals with highly controlled topology. Such substrates may exhibit very uniform surface

\author{
*Address correspondence to \\ piero.pompa@unile.it. \\ Received for review January 26, 2009 \\ and accepted March 13, 2009. \\ Published online March 25, 2009. \\ $10.1021 / \mathrm{nn} 900077$ s CCC: $\$ 40.75$ \\ (c) 2009 American Chemical Society
}



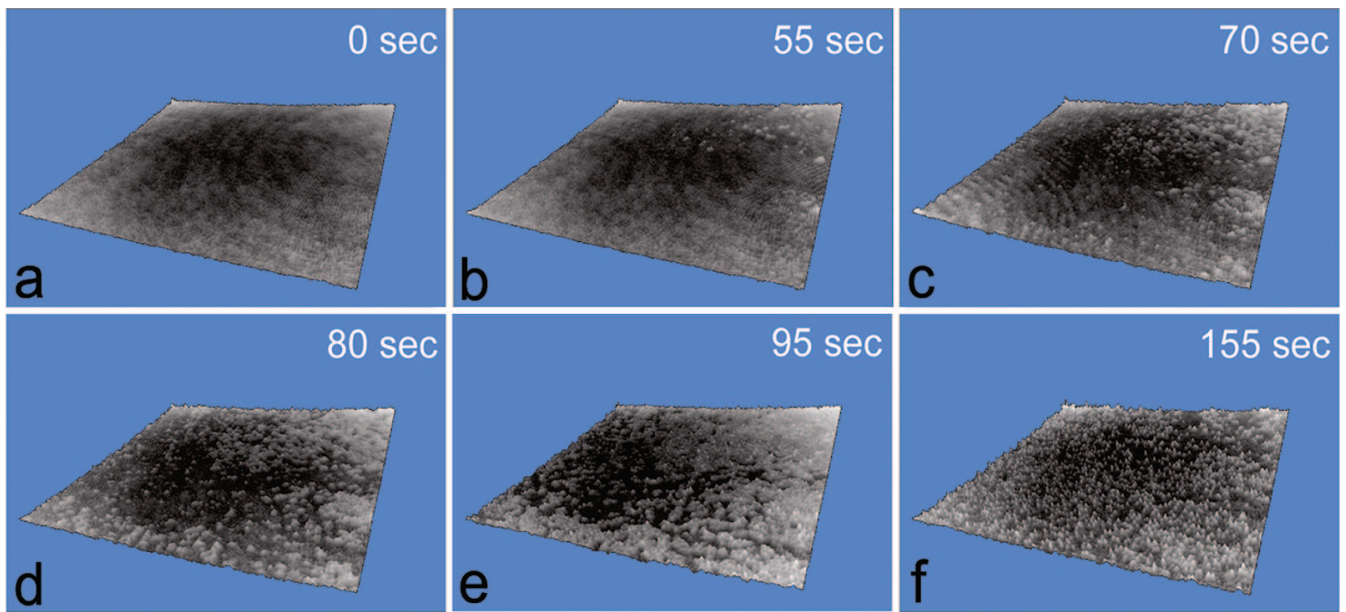

Figure 1. Real-time investigation of a SGDR reaction by holographic microscopy: a $15 \mathrm{~nm}$ thick silver film deposited onto an APTES-modified glass coverslip was displaced by gold. The images show the evolution of the SGDR reaction as a function time (indicated in each panel) until the reaction is completed (see the text for more details). The region analyzed in the figures is ca. $50 \mu \mathrm{m} \times 50 \mu \mathrm{m}$.

nanoroughness within desired regions of the pattern, leading to very uniform and efficient MEF effects throughout these selected regions (from submicron resolution to millimeter-sized substrates).

SGDR process is routinely used for electroless plating of metals in bulk materials and is also applied to make microcontacts in electronics industry. ${ }^{18}$ More recently, thick metal substrates roughened by SGDR processes have also been successfully applied for catalysis ${ }^{4}$ and surface-enhanced Raman spectroscopy (SERS). ${ }^{19}$ Buriak and co-workers have similarly reported on the deposition and patterning of metals using the SGDR process, though restricted only to semiconducting surfaces, such as silicon and GaAs. ${ }^{20,21}$ Additionally, they demonstrated the possibility to pattern two different metals by using the self-assembling properties of a block copolymer, selective to two different kinds of metal ions, although, in this latter case, the process is limited by the chemical/physical properties of the block copolymer. ${ }^{22}$ On the other hand, an interesting MEF process over a large substrate has been recently reported by Goldys et al., 15,23 by depositing a monolayer of gold nanoparticles followed by a secondary seeded growth of silver over them, although an accurate spatial control of the process on the micrometer scale as well as the possibility to use this protocol for patterning two different metals has not been demonstrated. Unlike such approaches, the method described in this paper is quite undemanding, versatile, and low-cost, as it can provide an effective tool to realize desired patterns of different metals on a variety of substrates. Importantly, under proper optimization, it may lead to the achievement of nanostructured substrates, uniform over extended areas, which are particularly suitable for practical/commercial MEF applications (e.g., optical sensing devices).

The strategy presented here is based on the deposition of a thin metal film on a substrate (such as silicon dioxide, quartz, glass) by thermal evaporation; the metal layer is then allowed to spontaneously react with an aqueous solution of metal ions, having suitably high reduction potential, that can replace the predeposited metal in regions defined by an intermediate lithographic step. By varying the experimental parameters at different stages of the SGDR-based method, it is possible to control the nanostructuring and composition of the metal. This method allows, therefore, the fabrication of micron and submicron patterns of two different metals with high spatial accuracy. Such a possibility makes it feasible to develop a new class of substrates with patterns of different plasmonic properties at micro- and subwavelength scales. ${ }^{24}$ Additionally, with suitable surface modification (e.g., by exploiting the specific surface chemistry of different metals), they could also serve as patterned substrates for superhydrophobicity, superhydrophilicity, ${ }^{25,26}$ and tissue engineering. ${ }^{1}$

\section{RESULTS AND DISCUSSION}

Initially, we carefully investigated by holographic microscopy the SGDR process over a thin silver film deposited onto a glass substrate by monitoring in realtime the displacement of the silver layer by gold ions, leading to the formation of a rough gold film. In this experiment, a $15 \mathrm{~nm}$ thick silver film, deposited by thermal evaporation onto an APTES-modified glass coverslip, was reacted with chloroauric acid over the sample stage of the microscope. The reaction was carried out by first immersing the sample in $2 \mathrm{~mL}$ of water in a small Petri dish and then injecting $80 \mu \mathrm{L}$ of $0.01 \mathrm{M} \mathrm{HAuCl}_{4}$ aqueous solution into it. After the injection, spontaneous galvanic reaction of the gold ions with the deposited metallic silver occurs, resulting in the deposition of reduced gold species in their metallic form replacing the simultaneously oxidized products, $\mathrm{Ag}^{+}$ions. During the progress of the reaction, as observed from the 
naked eye, the color of the metal film changed from shiny yellow to a nonreflecting purple-black color. Figure 1 shows representative holographic phase images of intermediate steps captured during the progress of the reaction over a $50 \mu \mathrm{m}$ $\times 50 \mu \mathrm{m}$ area. Figure 1a represents the image of the unreacted silver film ( $t=0 \mathrm{~s}$, namely, just after the injection of the gold ions solution), while Figure $1 \mathrm{f}$ shows the film after the galvanic reaction with gold ions ends. During the holographic measurements, upon addition of $\mathrm{HAuCl}_{4}$ solution, no changes were observed in the initial period ( $\sim 40-50 \mathrm{~s})$, likely due to the time required for diffusion of the concentrated gold ion solution into the aqueous medium (i.e., until gold ions reach the observation point). At the beginning of the reaction, few elevated spot-like features can be observed over the film (Figure 1b), which can be ascribed to the initial deposition of gold, as nucleation sites, on the film surface replacing the underlying silver. The process is, hence, clearly observed to occur by initial nucleation at several sites on the surface followed by growth around these nucleated sites, until the galvanic reaction ceases (Figure $1 c-f) .{ }^{27}$ It may be also noted that, while the emergence of initial nucleation sites progresses slowly, growth around these nucleated sites proceeds quite rapidly. The progress of the reaction can be better visualized from the video clip provided in the Supporting Information. Notably, by holographic microscopy, we were able to observe for the first time the galvanic displacement mechanism in real-time in a noninvasive manner, disclosing in detail the dynamics of nucleation and growth of the SGDR process. ${ }^{27}$ Moreover, by comparing the features observed in the metal-coated substrate before (Figure 1a) and after (Figure 1f) the SGDR process, it is possible to note that the composition of the metal film appears to be transformed from a uniform and flat surface to a substantially different film in terms of morphology, exhibiting quite uniform nanoscale roughness, as also probed by AFM measurements (see also below). Such peculiar morphological features of the SGDR-based films, along with the possibility to precisely control the metal nanostructure by properly adjusting the parameters of the reaction (and of the film deposition), represent a key point for several applications, including MEF and hydrophobic substrates.

Interestingly, as the process is initiated at the metal surface, the reaction can be confined to selected regions by masking the surface in a desired pattern. Hence, SGDR processes may lead to the fabrication of micro- and nanoscale patterns of two (or more than two) different metals with relative facility. As an illustration, we have realized patterns of alternating gold and silver stripes. A schematic representation of the process
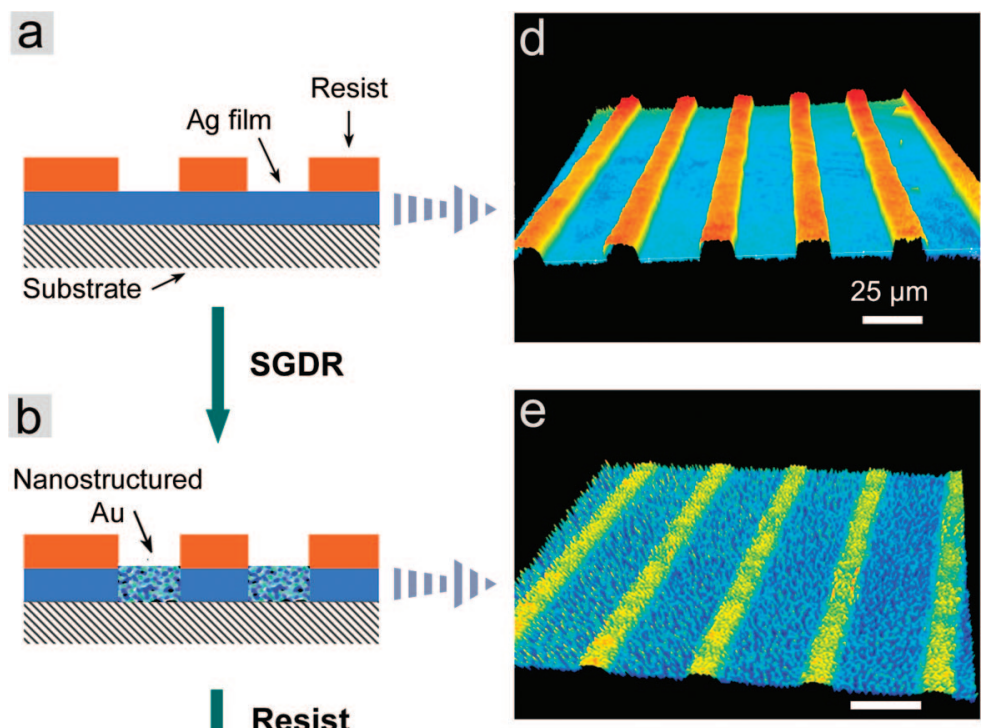

C

Resist removal

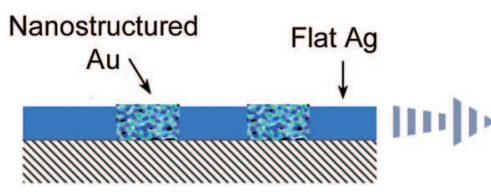

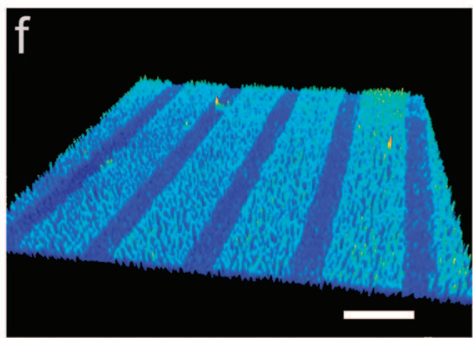

Figure 2. Localized SGDR process. (Left) schematic and (right) holographic microscopy images of the different steps of the procedure: (a) deposition of a resist mask layer onto a $15 \mathrm{~nm}$ thick silver film thermally evaporated onto an APTESmodified glass substrate; (b) localized galvanic displacement of silver by gold ions resulting in the formation of nanostructured gold stripes; (c) removal of the resist, showing the almost unperturbed flat morphology of the masked Ag regions. $(d-f)$ Holographic phase images of the corresponding steps represented in (a) - (c), respectively. The scale bars in all the holographic images are $25 \mu \mathrm{m}$ (the aspect ratio of the structure reported in the figure is very large, i.e., microns horizontally, nanometers vertically). The pattern shown in the right column was realized by optical lithography (the resist pattern was composed by $15 \mu \mathrm{m}$ wide stripes with a period of $40 \mu \mathrm{m}$ ).

to produce bimetallic patterns onto a substrate is reported in Figure $2 \mathrm{a}-\mathrm{c}$. A glass substrate, coated with a thin silver film, is first masked with a resist pattern using optical lithography; in the second step, the masked substrate is immersed in $3 \mathrm{~mL}$ of $10^{-4} \mathrm{M} \mathrm{HAuCl}_{4}$ aqueous solution for $30 \mathrm{~min}$ (such conditions were optimized for this patterned SGDR process). Finally, after the SGDR process is terminated, the resist layer is removed from the substrate. Figure $2 d-f$ are the representative 3D holographic phase images of the sample, corresponding to the three aforementioned steps. The resist layer consisted of $15 \mu \mathrm{m}$ wide stripes, masking the underlying silver film, with $25 \mu \mathrm{m}$ spacing (Figure 2d). After immersing the substrate in the $\mathrm{HAuCl}_{4}$ solution as mentioned above, features analogous to those reported in Figure $1 \mathrm{f}$ can be observed in the exposed regions (Figure 2e). Notably, subsequent removal of the resist layer resulted in a well-defined micrometer pattern of flat silver stripes with alternating rough gold stripes (Figure 2f). A lithographically patterned resist 
mask can be, therefore, exploited to successfully restrict the SGDR process only to the exposed area of a metal film. Moreover, since the patterned mask can be alternatively fabricated by electron beam lithography (EBL), any complex pattern with nanometer resolution can also be realized. In general, due to the overall simplicity of this strategy, the efficiency and reliability of the process was very good, with sample to sample reproducibility close to $100 \%$, in terms of spatial control of the pattern and uniformity of the surface roughness.

The versatility and efficiency of the procedure was demonstrated by realizing micro- and nanoscale gold/ silver bimetallic patterns onto APTES-modified $\mathrm{SiO}_{2}$ substrates, by using the SGDR method in combination with EBL technique. In these experiments, we fabricated two metal patterns of $2 \mu \mathrm{m}$ and $750 \mathrm{~nm}$ wide stripes. The surface morphology features were investigated by scanning electron microscopy (SEM). Unlike previous cases, in this pattern, the initially deposited metal layer was realized as a silver island film rather than a flat continuous film in order to further explore the potential of the SGDR process to fabricate patterned rough/plasmonic substrates. Such island-like structured film exhibited a yellowish color, at variance with the standard white mirror-like appearance of the continuous silver films. Representative SEM images of the pattern revealed well-defined $\mathrm{Au}$ and $\mathrm{Ag}$ parallel stripes (Figure 3). The observed structure of the silver regions is identical to that observed with SEM prior to the reaction, indicating efficient masking of the resist layer to the SGDR reaction. On the other hand, the exposed regions that underwent galvanic displacement with gold exhibited highly porous and rough nanostructures, grown out of the island-like template of the sacrificial silver layer. The ability to fabricate such patterns of two metals with differing nanostructures that are specifically confined into desired regions, with submicrometric resolution, may be of great interest for several applications, as mentioned above. Notably, these highly nanostructured metal substrates can act as spatially controlled regions for efficient electromagnetic field enhancement, in close proximity to their surface. ${ }^{28}$ Also, this approach allows the fabrication of patterns of a wide variety of metals, including both magnetic and nonmagnetic materials, such as platinum, copper, nickel, and cobalt (not shown).

Importantly, this strategy can be exploited for the realization of efficient and spatially controlled metalenhanced fluorescence substrates. In this latter case, a proper optimization of the metal surface, especially in terms of increased roughness, is required. To this purpose, we developed a tailored fabrication scheme based on the displacement of a thicker silver film (50 $\mathrm{nm}$ ) by a more concentrated gold ions solution $\left(10^{-2}-10^{-3} \mathrm{M} \mathrm{HAuCl}_{4}\right.$ aqueous solution) in $20 \mathrm{~min}$. This procedure results in the formation of localized rough gold regions, characterized by a significant uni-

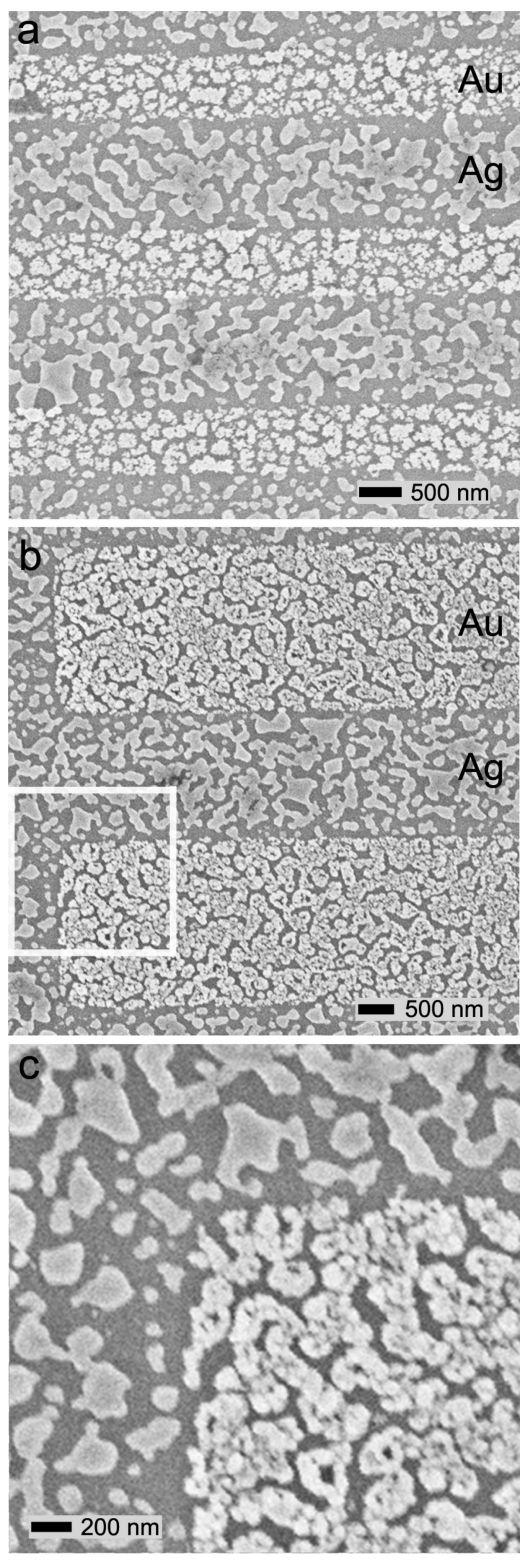

Figure 3. Scanning electron microscope images of nanoscale bimetallic patterns of silver and gold. A silver island film was selectively masked with an EBL-defined resist layer and subsequently immersed in $\mathrm{a} \mathrm{HACl}_{4}$ aqueous solution. Following resist removal, patterns of alternating stripes of nanostructured gold and silver of different dimensions were realized: (a) $750 \mathrm{~nm}$ wide gold stripes with a period of 2 $\mu \mathrm{m}$; (b) $2 \mu \mathrm{m}$ wide stripes with a period of $4 \mu \mathrm{m}$; (c) higher magnification image revealing the nanostructure of the gold within the area highlighted in image (b).

formity of the surface morphology, while substantially preventing diffusive reaction below the area masked by the resist layer. We tested the versatility of this process by realizing several bimetallic patterns of silver and gold (not shown), similar to those reported above, and patterns of flat and nanorough gold regions, specifically designed to better investigate fluorescence enhancement processes. Such substrates were realized by a localized deposition of $50 \mathrm{~nm}$ thick silver regions, by using optically patterned resist layers, onto a flat gold film, followed by SGDR displacement of silver by gold, as de- 
scribed above. Representative optical images of these substrates, with 20 $\mu \mathrm{m} \times 16 \mu \mathrm{m}$ silver islands (50 $\mathrm{nm}$ thickness), defined by bromography, over a $50 \mathrm{~nm}$ thick gold film, before and after the SGDR reaction, are shown in Figure 4a,b, respectively. Clearly, well-defined SGDR patterns were achieved also in these conditions: dark squares, replacing the sacrificial silver layers, can be observed. Such nanorough gold regions are distinctively separate from the surrounding flat gold film, giving mirror-like reflection. Remarkably, the same features can be also obtained with good accuracy on a very large substrate, such as the centimeter-sized gold pattern partially shown in the SEM image of Figure 4c. The morphology of these gold structures was assessed by atomic force microscopy (AFM). A representative 3D image of a rough gold square is reported in Figure $4 d$, clearly showing the significant surface roughness of the SGDR structure that is well-confined within the patterned region. Two typical AFM line profiles taken within such region revealed the presence of a quite uniform nanoscale roughness, with typical $100-150 \mathrm{~nm}$ peak-to-valley values (Figure 4e). Conversely, the surface roughness exhibited by the flat gold film was in the $1-2 \mathrm{~nm}$ range.

Such gold nanostructures were demonstrated to be highly efficient for metal-enhanced fluorescence processes. We investigated the relative effects of flat and nanorough gold patterns on a model fluorophore placed in close proximity to the metal surfaces under otherwise identical conditions. Fluorescein isothiocyanate (FITC) molecules were covalently and uniformly bound to these patterned substrates by using cysteine molecules as intermediate linkers (see Experimental Section for details). Upon immersion of the gold samples in cysteine solutions, cysteine molecules bind to the gold surface preferably through the thiol group, forming the gold-sulfur bond ${ }^{29}$ and leaving the amine groups free to covalently link with the FITC molecules. ${ }^{30}$ Using this method, FITC-covered gold substrates with surface roughness patterned in the form of $125 \mu \mathrm{m}$ wide stripes and of an array with $20 \mu \mathrm{m}$ features were fabricated. Representative confocal fluorescence images of such FITC gold-patterned substrates are shown in Figure 5a,b, revealing highly enhanced emission of the fluorophore from the patterned nanorough gold regions. Such increased fluorescence intensity is due to
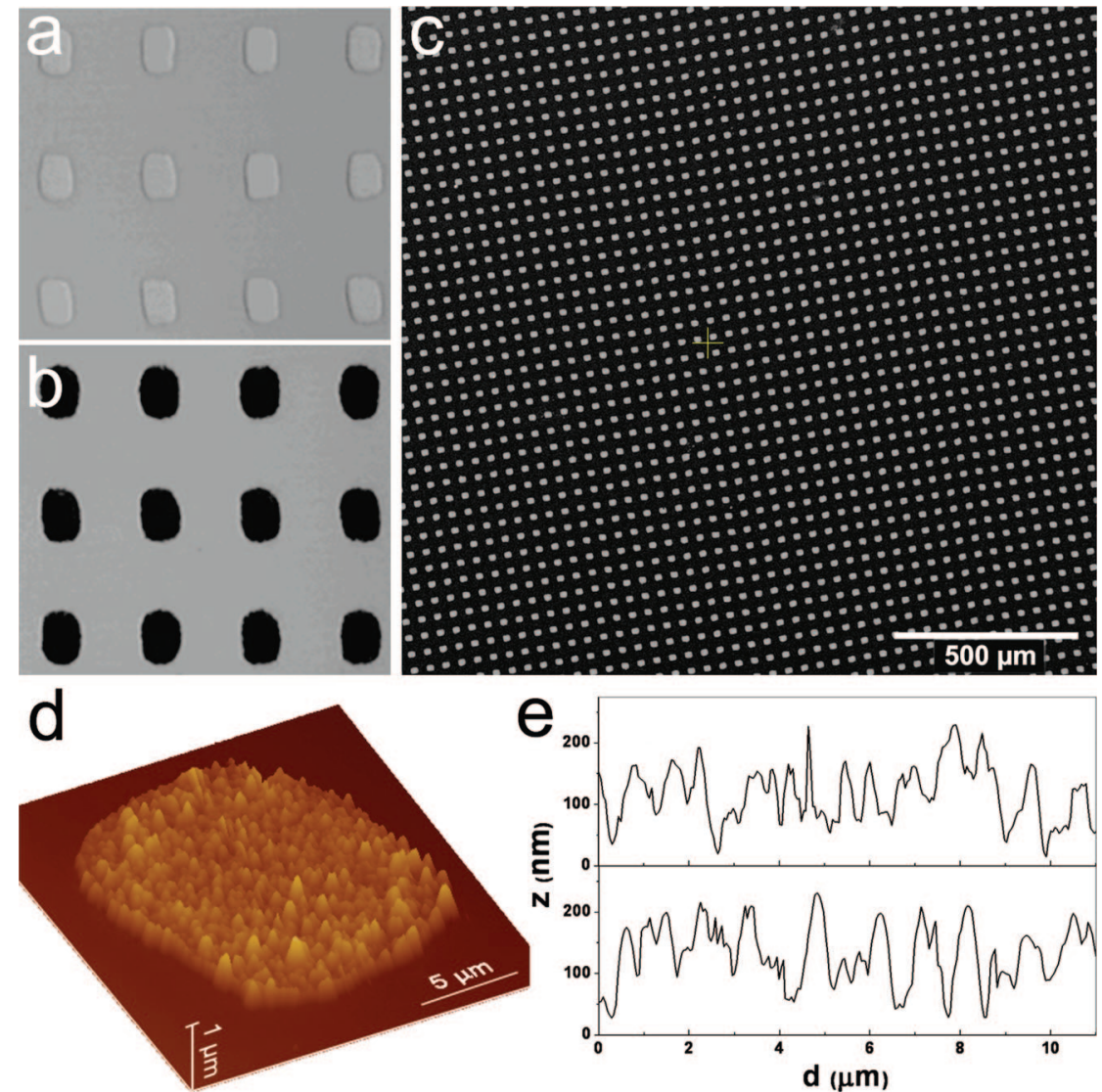

Figure 4. Representative patterns of rough gold regions onto flat gold films. (a) Optical imout the garryof a typical nanorough gold region; (e) two representative line profiles taken within the rough

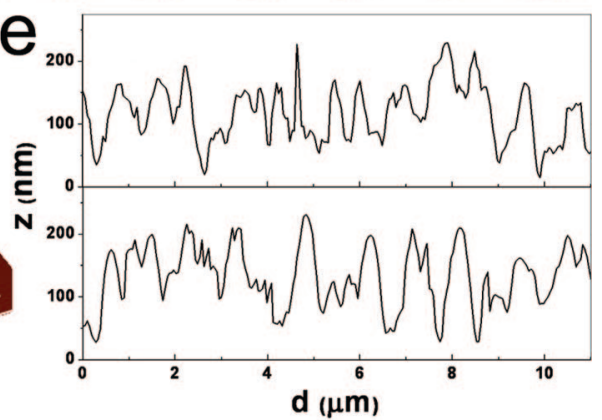

(1)

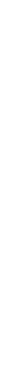

the coupling of the fluorophore with the surface plasmons (SPs) of the underlying rough gold film, resulting in a remarkable increase of the FITC excitation rate (see below). Interestingly, both images show a very good uniformity of the enhanced emission over large areas, very intense fluorescence signals, along with a precise spatial control of the MEF process (see also the fluorescence line profiles reported in Figure $5 c$,d). Notably, from the fluorescence profiles, it is possible to estimate a $\sim 50$-fold fluorescence increase of the SPs-coupled FITC with respect to the fluorophore positioned onto the flat metal film. Such intensity enhancement can be controlled with good uniformity both at the millimeter scale (Figure $5 a, b)$ and on the micro- and submicrometer scale, as demonstrated by the bright rough gold island, defined by E-beam lithography, reported in Figure $5 \mathrm{~d}$. The increased surface area of the rough regions gives a negligible contribution $(\sim 0.1 \%)$ to the observed fluorescence enhancement. In fact, since the roughness of our plasmonic substrates is in the nanometer range, the overall increase of the surface area of the enhancing regions is very low (only $10-20 \%$ higher than the flat gold surface, as calculated from AFM images). 


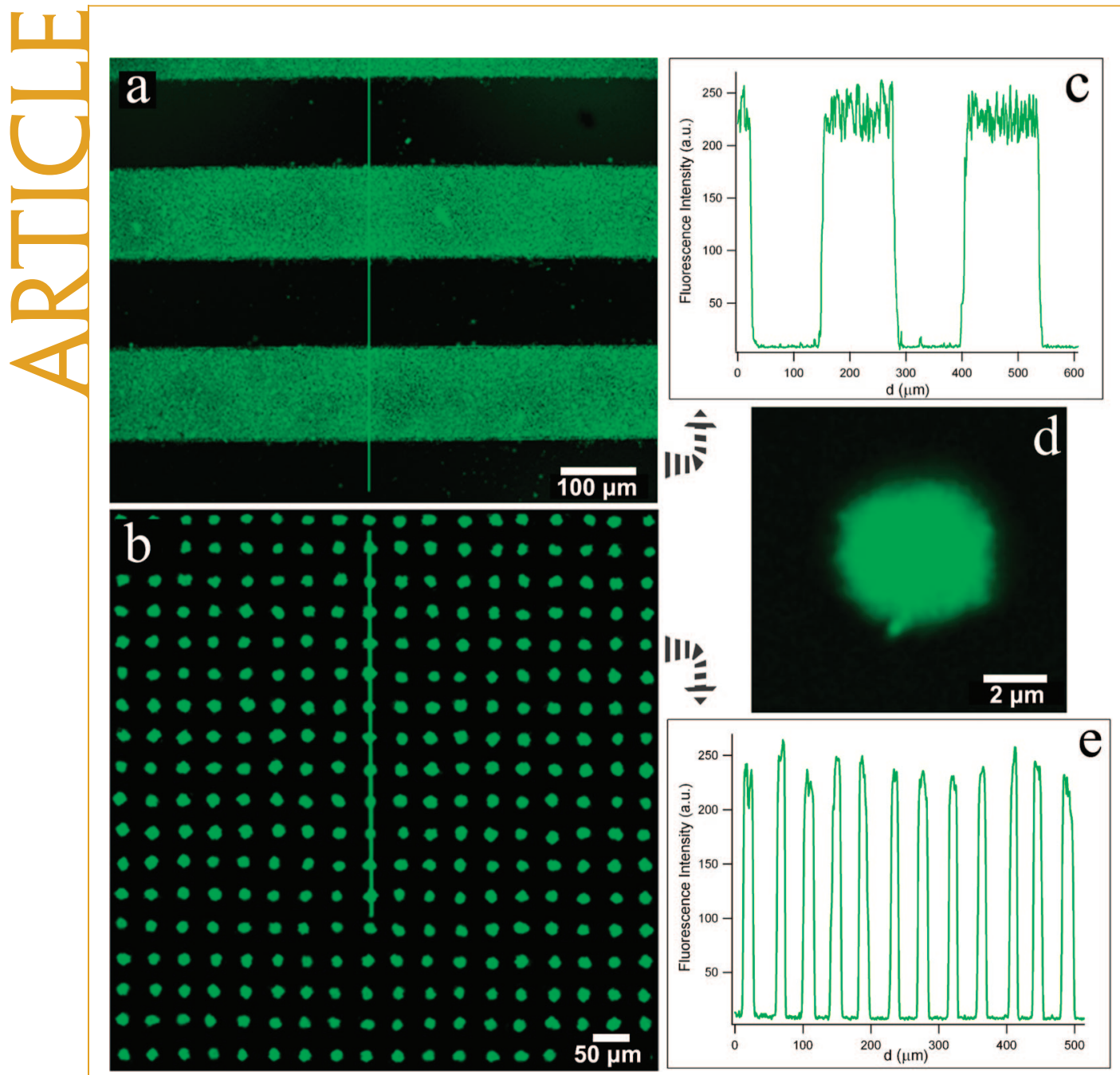

Figure 5. Spatially resolved fluorescence images of fluorescein immobilized onto the gold patterns taken by confocal microscopy. A clear MEF increase of FITC emission intensity can be observed in correspondence to the nanostructured gold regions: (a) $125 \mu \mathrm{m}$ wide rough gold stripes onto a flat gold film; (b) array with $20 \mu \mathrm{m}$ rough gold features; (d) a single rough gold island $(\sim 4 \mu \mathrm{m})$ defined by E-beam lithography; $(c, e)$ fluorescence line profiles taken over the images reported in (a) and (b), respectively.

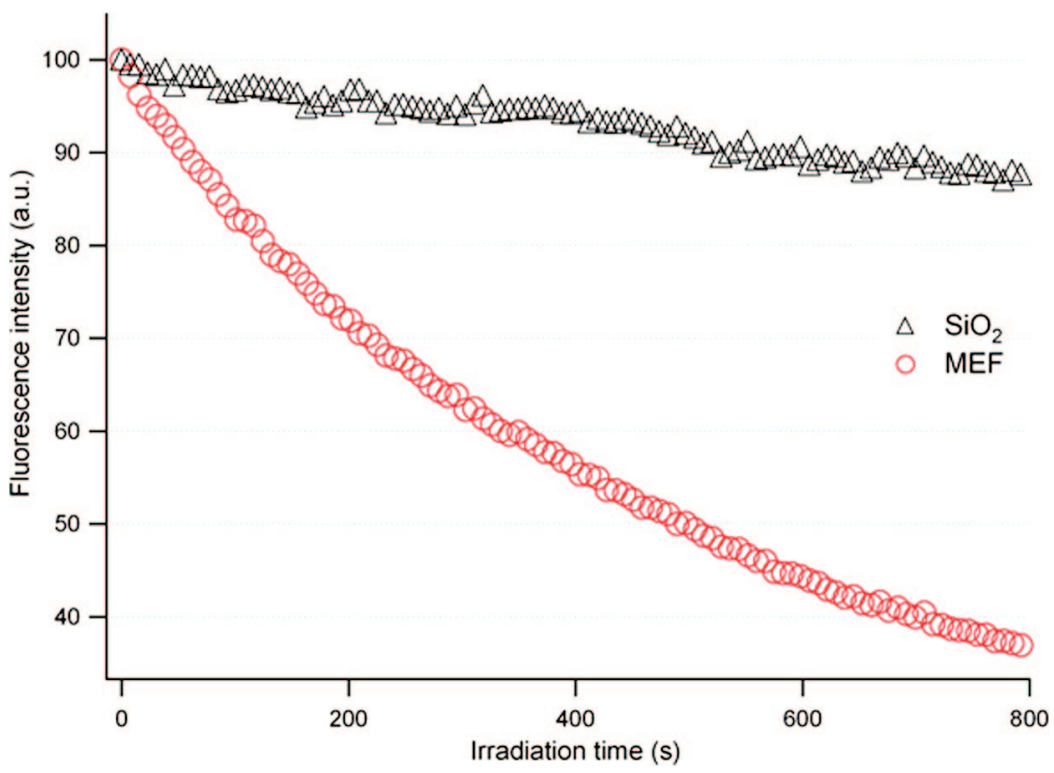

Figure 6. Photobleaching rate of SPs-coupled FITC (red circles) as compared to that of FITC bound to a reference APTES-modified silicon dioxide substrate (black triangles) upon prolonged high-intensity irradiation.
The FITC fluorescence enhancement by the nanostructured gold regions was also assessed in comparison to standard reference substrates such as silica or glass since significant quenching of the fluorophore emission can be expected onto flat gold films. ${ }^{7,8}$ We thus fabricated the same nanorough gold pattern onto a large APTES-modified silica substrate (onto which FITC molecules bind via the exposed amino groups), evaluating an enhancement factor of our plasmonic substrates of $c a$. 15 -fold with respect to silica or glass (i.e., in our system, the flat gold surface induces an emission quenching higher than 3-fold as compared to silica). Considering the high quantum yield of fluorescein $(\sim 0.9)$, it is very likely that the overall fluorescence enhancement we observed in FITC is mostly due to an increase of its excitation rate elicited by a large increase in the local density of the excitation field, in close proximity to the rough gold surface. ${ }^{7,28}$ Also, spectral analyses revealed that the

fluorescence line shape of the MEF-increased FITC emission was very similar to that of the reference fluorophore, with no detectable modifications/shifts caused by the interaction with gold plasmons. ${ }^{7}$ In any case, we verified this point by comparing the photobleaching rate of the FITC molecules, localized both onto the rough gold and onto the reference silica substrate, upon prolonged exposure to high intensity radiation. We observed that the FITC localized onto the gold substrate underwent a significantly faster photobleaching as compared to the fluorescein on silica (under otherwise identical conditions; Figure 6). This means that the enhancement mechanism is largely caused by an increase in the excitation rate of FITC molecules (i.e., SPs-coupled fluorescein undergoes a higher number of excitation/emission cycles per time unit with respect to the reference fluorophores), resulting in an overall enhancement of the emission inten- 
sity. ${ }^{7}$ Interestingly, the slope of the photobleaching curve of the MEF fluorophore in its initial part was calculated to be 13.6-fold higher than that of FITC immobilized onto $\mathrm{SiO}_{2}$, in close agreement with the overall enhancement factor $(\sim 15)$ observed onto the our plasmonic substrates. Moreover, time-resolved fluorescence measurements performed onto silica and rough gold revealed similar lifetimes for FITC, suggesting the absence of significant perturbations in the emission rate of the fluorophore. This further confirms our interpretation of the enhancement mechanism.

\section{CONCLUSIONS}

We have shown that our SGDR-based process can serve as a versatile, low-cost, and very efficient method to fabricate micro- and nanoscale patterns of different metals and/or highly nanostructured metal surfaces. The feasibility of fabricating these films over a number of suitable substrates (such as silica, glass, quartz, and even plastic) opens attractive perspectives for a wide range of applications, including MEF, SERS, solar cells, DNA microarray technology, hydrophobic/hydrophilic substrates, tissue engineering, and magnetic devices. Additionally, these substrates may be further developed to produce a new class of materials to investigate the interactions between cells and nanostructured surfaces or to study interesting optical properties with patterned features in the subwavelength range.

\section{EXPERIMENTAL SECTION}

Reagents. Aminopropyltriethoxysilane (APTES), L-cysteine, and $\mathrm{HAuCl}_{4}$ were purchased from Sigma Aldrich. Ammonium hydroxide (30\%), hydrogen peroxide (30\%), hydrochloric acid (37\%), acetone, and isopropanol were purchased from JT Baker. Microposit primer (Anira), AZ 5214E positive resist, and AZ 726MIF developer solutions were obtained from Clarian. PMMA 950K resist and MIBK developer solution were purchased from Allresist $\mathrm{GmbH}$. Deionized water (resistivity, $18.2 \mathrm{M} \Omega \cdot \mathrm{cm}$ ) was used for preparation of all aqueous solutions and washing of substrates.

Fabrication and Characterization. For the preparation of the patterned samples, the substrates, either glass slides or silica, were first treated with a 1:1:5 ratio solution of $30 \% \mathrm{NH}_{3} \mathrm{OH}, 30 \% \mathrm{H}_{2} \mathrm{O}_{2}$, and water at $80{ }^{\circ} \mathrm{C}$ for $10 \mathrm{~min}$ followed by treatment with 1:1:5 ratio solution of $30 \% \mathrm{HCl}, 30 \% \mathrm{H}_{2} \mathrm{O}_{2}$, and water at $80{ }^{\circ} \mathrm{C}$ for 10 min and intermediate steps of washing with deionized water after each treatment. Subsequently, the cleaned substrates were immersed in $0.5 \%$ APTES aqueous solution for 5 min, washed with deionized water, and kept in vacuum overnight. To fabricate a two-metal pattern, the APTES-modified substrates were coated with silver thin film of desired thickness using thermal vacuum deposition. These substrates were subsequently spin coated with AZ resist or PMMA for further lithography using bromography or EBL, respectively. After exposure and development of the resist pattern, the substrates were treated with $\mathrm{HAuCl}_{4}$ aqueous solutions, according to the experimental conditions (i.e., molarity and time of incubation) as described in the text for each case. Finally, these substrates were sequentially rinsed in hot acetone and isopropanol for $10 \mathrm{~min}$ each, followed by drying under high purity nitrogen flow, to completely remove the resist layer. For the fabrication of patterned substrates with single metal but different surface roughness, APTESmodified substrates were first coated with very flat $50 \mathrm{~nm}$ thick gold film by thermal vacuum deposition. These substrates were then masked with a patterned resist layer and subsequently coated with $50 \mathrm{~nm}$ thick silver films, followed by the resist removal as described above. These substrates were reacted by immersing them in $\mathrm{HAuCl}_{4}$ aqueous solutions followed by washing in deionized water twice for 5 min each and drying under high purity nitrogen flow. This step leads to the formation of a pattern of nanostructured gold islands, replacing the silvercoated regions, onto a flat gold film. All these substrates were characterized by holographic microscopy (Lyncèe Tec DHM 1000, transmission mode), AFM (Nanoscope IV MultiMode, Veeco Instruments), and SEM (Nova NanoSEM200, FEI).

For MEF experiments, these substrates were incubated overnight in $10^{-3} \mathrm{M}$ cysteine solution, prepared with degassed deionized water. Later, after thoroughly washing with deionized water and drying with flowing nitrogen, these cysteine-modified substrates were incubated with $0.001 \%$ FITC solution at $0{ }^{\circ} \mathrm{C}$ for $4 \mathrm{~h}$. This step leads to the covalent binding of FITC to the free amine group of cysteine molecules bound to the gold surface through their thiol group. Finally, these substrates were thoroughly washed with deionized water and dried in flowing nitrogen to wash out any noncovalently bound FITC molecules. These FITC-modified substrates were then studied using confocal microscope (Leica TCS-SP5 AOBS). The excitation wavelength $\left(\lambda_{\text {exc }}\right)$ was typically 458 or $488 \mathrm{~nm}$ (no significant differences were observed); samples were observed through a 20X, 0.70 NA objective or a $63 \mathrm{X}, 1.40 \mathrm{NA}$ oil immersion objective. For spectral analysis, $\lambda_{\text {exc }}$ was 458 or $488 \mathrm{~nm}$, and the emission bandwidth was 10 $\mathrm{nm}$.

Acknowledgment. The authors gratefully acknowledge $\mathrm{S}$. Sabella, V. Brunetti, G. Vecchio, B. Sorce, A. Della Torre, and L. Martiradonna for help during experiments, and E. D'Amone and V. Fiorelli for the expert technical assistance. This work was supported by the Italian Ministry of Research through MIUR "FIRB" project (RBLA03ER38_001).

Supporting Information Available: Real-time imaging by holographic microscopy of the SGDR deposition of a nanorough gold film replacing a flat silver layer on a glass substrate (the region analyzed in the video clip is ca. $50 \mu \mathrm{m} \times 50 \mu \mathrm{m})$. This material is available free of charge via the Internet at http://pubs.acs.org.

\section{REFERENCES AND NOTES}

1. Khang, D.; Lu, J.; Yao, C.; Haberstroh, K. M.; Webster, T. J. The Role of Nanometer and Sub-Micron Surface Features on Vascular and Bone Cell Adhesion on Titanium. Biomaterials 2008, 29, 970.

2. Bonroy, K.; Friedt, J.-M.; Frederix, F.; Laureyn, W.; Langerock, S.; Campitelli, A.; Sara, M.; Borghs, G.; Goddeeris, B.; Declerck, P. Realization and Characterization of Porous Gold for Increased Protein Coverage on Acoustic Sensors. Anal. Chem. 2004, 76, 4299.

3. Kramer, D.; Viswanath, R. N.; Weissmüller, J. Surface-Stress Induced Macroscopic Bending of Nanoporous Gold Cantilevers. Nano Lett. 2004, 4, 793.

4. Bansal, V.; Jani, H.; Plessis, J. D.; Coloe, P. J.; Bhargava, S. K. Galvanic Replacement Reaction on Metal Films: a OneStep Approach to Create Nanoporous Surfaces for Catalysis. Adv. Mater. 2008, 20, 717.

5. Namavar, F.; Cheung, C. L.; Sabirianov, R. F.; Mei, W.-N.; Zeng, X. C.; Wang, G.; Haider, H.; Garvin, K. L. Lotus Effect in Engineered Zirconia. Nano Lett. 2008, 8, 988.

6. Shi, F.; Song, Y.; Niu, J.; Xia, X.; Wang, Z.; Zhang, X. Facile Method to Fabricate a Large-Scale Superhydrophobic Surface by Galvanic Cell Reaction. Chem. Mater. 2006, 18, 1365.

7. Lakowicz, J. R. Radiative Decay Engineering: Biophysical and Biomedical Applications. Anal. Biochem. 2001, 298, 1. 
8. Fort, E.; Grésillon, S. Surface Enhanced Fluorescence. J. Phys. D: Appl. Phys. 2008, 41, 013001.

9. Aslan, K.; Gryczynski, I.; Malicka, J.; Matveeva, E.; Lakowicz, J. R.; Geddes, C. D. Metal-Enhanced Fluorescence: An Emerging Tool in Biotechnology. Curr. Opin. Biotechnol. 2005, 16, 55.

10. Tvingstedt, K.; Persson, N. K.; Inganäs, O.; Rahachou, A.; Zozoulenko, I. V. Surface Plasmon Increase Absorption in Polymer Photovoltaic Cells. Appl. Phys. Lett. 2007, 91, 113514.

11. Lakowicz, J. R.; Shen, Y.; D’Auria, S.; Malicka, J.; Fang, J.; Gryczynski, Z.; Gryczynski, I. Radiative Decay Engineering 2. Effects of Silver Island Films on Fluorescence Intensity, Lifetimes, and Resonance Energy Transfer. Anal. Biochem. 2002, 301, 261.

12. Geddes, C. D.; Parfenov, A.; Lakowicz, J. R. Photodeposition of Silver Can Result in Metal-Enhanced Fluorescence. Appl. Spectrosc. 2003, 57, 526.

13. Geddes, C. D.; Parfenov, A.; Roll, D.; Fang, J.; Lakowicz, J. R. Electrochemical and Laser Deposition of Silver for Use in Metal-Enhanced Fluorescence. Langmuir 2003, 19, 6236.

14. Geddes, C. D.; Parfenov, A.; Roll, D.; Gryczynski, I.; Malicka, J.; Lakowicz, J. R. Roughened Silver Electrodes for Use in Metal-Enhanced Fluorescence. Spectrochim. Acta, Part A 2004, 60, 1977.

15. Xie, F.; Baker, M. S.; Goldys, E. M. Enhanced Fluorescence Detection on Homogeneous Gold Colloid Self-Assembled Monolayer Substrates. Chem. Mater. 2008, 20, 1788.

16. Ahl, S.; Cameron, P. J.; Liu, J.; Knoll, W.; Erlebacher, J.; Yu, F. A Comparative Plasmonic Study of Nanoporous and Evaporated Gold Films. Plasmonics 2008, 3, 13.

17. Pompa, P. P.; Martiradonna, L.; Della Torre, A.; Della Sala, F.; Manna, L.; De Vittorio, M.; Calabi, F.; Cingolani, R.; Rinaldi, R. Metal Enhanced Fluorescence of Colloidal Nanocrystals with Nanoscale Control. Nat. Nanotechnol. 2006, 1, 126.

18. Green, T. A. Gold Electrodeposition for Microelectronic, Optoelectronic and Microsystem Applications. Gold Bull. 2007, 40, 105

19. Wang, C.-H.; Sun, D.-C.; Xia, X.-H. One-Step Formation of Nanostructured Gold Layers via a Galvanic Exchange Reaction for Surface Enhancement Raman Scattering. Nanotechnology 2006, 17, 651.

20. Porter, L. A., Jr.; Choi, H. C.; Schmeltzer, J. M.; Ribbe, A. E.; Elliott, L. C. C.; Buriak, J. M. Electroless Nanoparticle Film Deposition Compatible with Photolithography, Microcontact Printing, and Dip-Pen Nanolithography Patterning Technologies. Nano Lett. 2002, 2, 1369.

21. Nezhad, M. R. H.; Aizawa, M.; Porter, L. A., Jr.; Ribbe, A. E.; Buriak, J. M. Synthesis and Patterning of Gold Nanostructures on InP and GaAs via Galvanic Displacement. Small 2005, 1, 1076.

22. Aizawa, M.; Buriak, J. M. Nanoscale Patterning of Two Metals on Silicon Surfaces Using an ABC Triblock Copolymer Template. J. Am. Chem. Soc. 2006, 128, 5877.

23. Xie, F.; Baker, M. S.; Goldys, E. M. Homogeneous SilverCoated Nanoparticle Substrates for Enhanced Fluorescence Detection. J. Phys. Chem. B 2006, 110, 23085.

24. Giessen, H. Grating Games. Nat. Photonics 2008, 2, 335.

25. Zhai, L.; Berg, M. C.; Cebeci, F. C.; Kim, Y.; Milwid, J. M.; Rubner, M. F.; Cohen, R. E. Patterned Superhydrophobic Surfaces: Toward a Synthetic Mimic of the Namib Desert Beetle. Nano Lett. 2006, 6, 1213-1217.

26. Gau, H.; Herminghaus, S.; Lenz, P.; Lipowsky, R. Liquid Morphologies on Structured Surfaces: from Microchannels to Microchips. Science 1999, 283, 46.

27. Porter, L. A., Jr.; Choi, H. C.; Ribbe, A. E.; Buriak, J. M Controlled Electroless Deposition of Noble Metal Nanoparticle Films on Germanium Surfaces. Nano Lett. 2002, 2, 1067.

28. Bozhevolnyi, S. I.; Beermann, J.; Coello, V. Direct Observation of Localized Second-Harmonic Enhancement in Random Metal Nanostructures. Phys. Rev. Lett. 2003, 90, 197403.
29. Uvdal, K.; Bodö, P.; Liedberg, B. L-Cysteine Adsorbed on Gold and Copper: An X-ray Photoelectron Spectroscopy Study. J. Colloid Interface Sci. 1992, 149, 162.

30. Jobbagy, A.; Kiraly, A. Chemical Characterization of Fluorescein Isothiocyanate-Protein Conjugates. Biochim. Biophys. Acta 1966, 124, 166. 